Review Article

\title{
Overcoming P-Glycoprotein-Mediated Multidrug Resistance in Colorectal Cancer: Potential Reversal Agents among Herbal Medicines
}

\author{
Gayoung Lee $\mathbb{D},{ }^{1,2,3}$ Jin-Yong Joung $\mathbb{D},{ }^{2,3}$ Jung-Hyo Cho $\mathbb{D},{ }^{2,3}$ \\ Chang-Gue Son $\left(\mathbb{D},,^{2,3}\right.$ and Namhun Lee $\mathbb{D}^{1,2,3}$ \\ ${ }^{1}$ Department of Clinical Oncology, Cheonan Korean Medicine Hospital of Daejeon University, 4, Notaesan-ro, Seobuk-gu, \\ Cheonan-si, No. 31099, Republic of Korea \\ ${ }^{2}$ Liver and Immunology Research Center, Dunsan Korean Medicine Hospital of Daejeon University, 75, \\ Daedeok-daero 176beon-gil, Seo-gu, Daejeon-si, No. 35235, Republic of Korea \\ ${ }^{3}$ Department of Internal Medicine, Graduated School of Korean Medicine, University of Daejeon, 62, Daehak-ro, \\ Dong-gu, Daejeon-si, No. 34520, Republic of Korea
}

Correspondence should be addressed to Namhun Lee; nhlee@dju.kr

Received 17 April 2018; Revised 24 July 2018; Accepted 2 August 2018; Published 12 August 2018

Academic Editor: María C. Carpinella

Copyright (C) 2018 Gayoung Lee et al. This is an open access article distributed under the Creative Commons Attribution License, which permits unrestricted use, distribution, and reproduction in any medium, provided the original work is properly cited.

Objectives. Multidrug resistance (MDR) is the major reason for the failure of chemotherapy in colorectal cancer (CRC), and the primary determinant of MDR in CRC patients is active drug efflux owing to overexpression of P-glycoprotein (P-gp) in cancer tissues. Despite research efforts to overcome P-gp-mediated drug efflux, the high toxicity of P-gp inhibitors has been a major obstacle for the clinical use of these agents. The aim of this study was to review the literature for potential P-gp reversal agents among traditional herbal medicines, which offer the advantages of safety and potential synergetic effects in CRC chemotherapy. Methods. We searched ten databases including 3 English databases, 1 Chinese medical database, and 6 Korean medical databases up to July 2018 and included in vivo and in vitro studies evaluating the effects of herbal medicines as P-gp reversal agents in CRC. Results. A total of 28 potentially related studies were identified and 16 articles were included. Involving 3 studies about Salvia miltiorrhiza and 2 studies about Curcuma longa, finally we found 14 kinds of traditional herbal medicines-Salvia miltiorrhiza, Curcuma longa, Sinomenium acutum, Stephania tetrandra, Bufo gargarizans, Coptis japonica, Piper nigrum and Piper longum, Hedyotis diffusa, Schisandra chinensis, Glycyrrhiza glabra, Glycyrrhiza inflate, Daphne genkwa, Stemona tuberosa Lour, and Andrographis paniculata-as showing efficacy as P-gp inhibitors in anticancer drug-resistant CRC cells in vitro and in vivo. Conclusions. This brief account provides insight into the relationship between P-gp and CRC. Further studies on herbal medicines with demonstrated effects against P-gp overexpression will aid in improving the efficacy of chemotherapy in CRC.

\section{Introduction}

Colorectal cancer (CRC) is the third most common cancer in the United States and over 50,000 deaths from CRC occurred in 2017 in the United States. [1]. Chemotherapy is regarded as an important treatment for CRC. However, the development of drug resistance usually results in the failure of chemotherapy in advanced CRC $[2,3]$. Several mechanisms of resistance have been identified to date, including increased drug efflux, reduced drug intake, cell cycle disruptions, alterations in the drug target, and sequestration of drugs $[4,5]$. In particular, 'active drug efflux' by overexpressed ATP-binding cassette $(\mathrm{ABC})$ transporters, which causes alterations in the intracellular accumulation of drug and thereby diminishes the efficacy of chemotherapy $[6,7]$, is cited as a common mechanism.

The P-glycoprotein (P-gp) transporter, referred to as ABCB1 (ATP-binding cassette subfamily $B$ member 1 ) or 
MDR1 (multidrug resistance protein 1), is among the most clinically important $\mathrm{ABC}$ transporters in the context of the gastrointestinal system $[3,8]$. P-gp is physiologically expressed in normal cells of the adrenal gland, pancreas, liver, brain, and intestines. In the human gastrointestinal tract, Pgp is highly concentrated on the apical surfaces of superficial columnar epithelial cells of the colon [9-11]. However, P-gp is pathologically associated with colorectal carcinogenesis and tumor grade $[12,13]$. Since Juliano and Ling first discovered the role of P-gp in drug-resistant cells in 1976, the structure of P-gp has been extensively studied, with several models demonstrating the drug export mechanism of P-gp [14]. P-gp consists of a single polypeptide with two NBDs (nucleotide binding domains) and two TMDs (transmembrane domains), each of which contains six transmembrane helices (TMH). This structure allows the formation of a binding pocket, which determines the substrate specificity of P-gp [15].

Investigations of strategies for overcoming P-gpmediated drug efflux during the last four decades have led to the development of several generations of P-gp inhibitors. However, the first three generations of P-gp inhibitors have shown disappointing results in humans because of the toxicity of these resistance-modifying agents [16]. Efforts to develop safer and more effective P-gp inhibitors have led researchers to consider plant-derived materials. Several researchers have demonstrated that alkaloids, flavonoids, and other plant compounds act as P-gp inhibitors when accompanied by chemotherapeutic drugs [17, 18]. Despite this large body of literature, no overviews of herbal medicines that act as P-gp reversal agents in CRC have been published. The present brief review has the goal of proposing the potential herbal medicines as P-gp reversal agents in CRC.

\section{Methods}

The following electronic databases were searched for studies from its inception to July 2018: English language databases (PubMed, EMBASE, and CINAHL), a Chinese literature database (China Academic Journal), and six Korean language databases (Korea Med, Oriental Medicine Advanced Medical Database (KM base), the Research Information Service System (RISS), National Digital Science Library, ProQuest Dissertations and Thesis, and Korean Studies Information Services System (KISS)). Studies in each language were screened using the following inclusion criteria: (1) in vitro or in vivo experiments, (2) effects at P-gp mediated MDR in colorectal cancer, and (3) subjects used as herbal medicines. In addition, the reference lists of potentially eligible articles were searched manually to identify additional relevant studies. An initial assessment was made by reading abstracts using the inclusion criteria. Then, articles meeting the inclusion criteria were read in full and we extracted data, including scientific name, herbal constituents, anticancer drug used, and pharmacological outcomes.

\section{Results}

Our literature searches revealed potentially 28 relevant articles, of which 16 met our inclusion criteria (Figure 1). 16

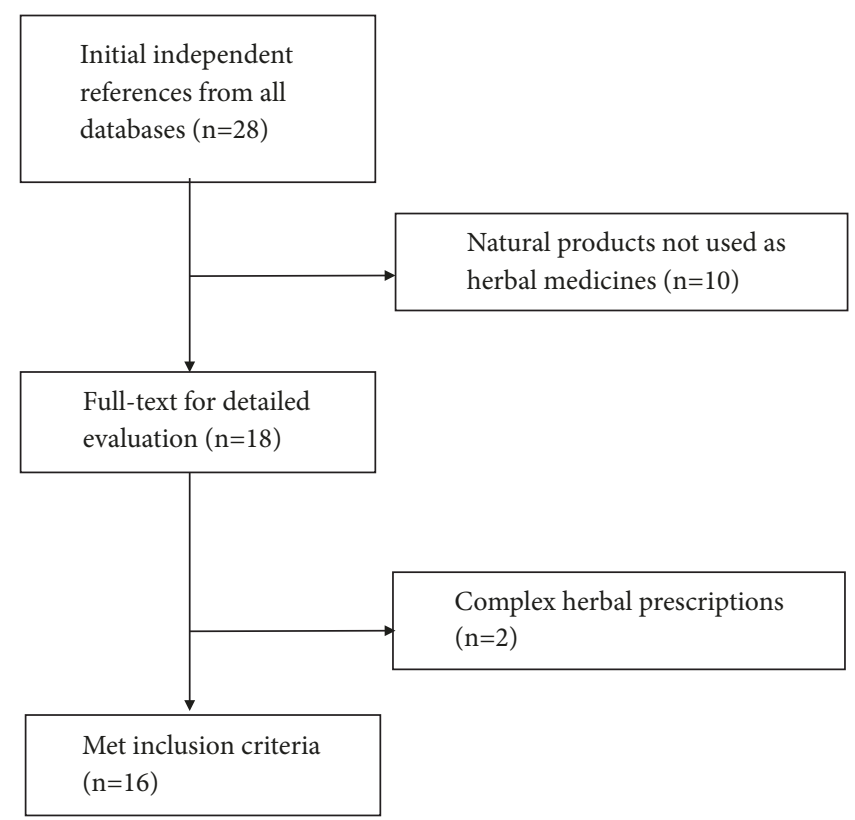

FIGURE 1: Scheme of the data selection process.

studies were conducted in vivo, and two studies using $C$. longa and $H$. Diffusa were both conducted in vivo and vitro. To examine the intestinal drug absorption in CRC, 7 studies used Caco- 2 cells, 3 used HCT- 8 cells, 2 used HCT-15 cells, and 1 each used THC- 8307, SW480 cell, and rat jejunum membranes. One study used LoVo/ADR, HCT116/L, and Caco-2/ADR cell lines.

Of the 16 studies, 3 studies were conducted using different herbal constituents extracted from Salvia miltiorrhiza and 2 studies were designed to evaluate curcumin with different ways. Finally, a total of 14 kinds of herbal medicines-Salvia miltiorrhiza, Curcuma longa, Sinomenium acutum, Stephania tetrandra, Bufo gargarizans, Coptis japonica, Piper nigrum and Piper longum, Hedyotis diffusa, Schisandra chinensis, Glycyrrhiza glabra, Glycyrrhiza inflate, Daphne genkwa, Stemona tuberosa Lour, Andrographis paniculata-can be credited with the potential to reverse P-gp-mediated multidrug resistance in specific drug-resistant CRCs. All herbal constituents, including their pharmacological effects observed experimentally, are briefly described below and summarized in Table 1.

3.1. S. miltiorrhiza Radix. The radix of S. miltiorrhiza (Chinese name, Danshen) has been traditionally used for the prevention and treatment of cardiovascular diseases. $S$. miltiorrhiza extracts contain tanshinones (I, IIA, and IIB), cryptotanshinone, tanshinol (I and II), and salviol, among other compounds [19]. Respectively, Tanshinone IIA seemed to be the substrate of P-gp, considering the decreased transport of digoxin in P-gp overexpressing membrane, and low oral bioavailability is explained by the first-pass metabolism [20]. Tanshinone IIB showed the inhibition of uptake of digoxin and vinblastine in P-gp or MRP1 membrane vesicles [35]. Hu et al. evaluated the inhibitory effects of five tanshinones (tanshinone I, tanshinone IIA, cryptotanshinone, 


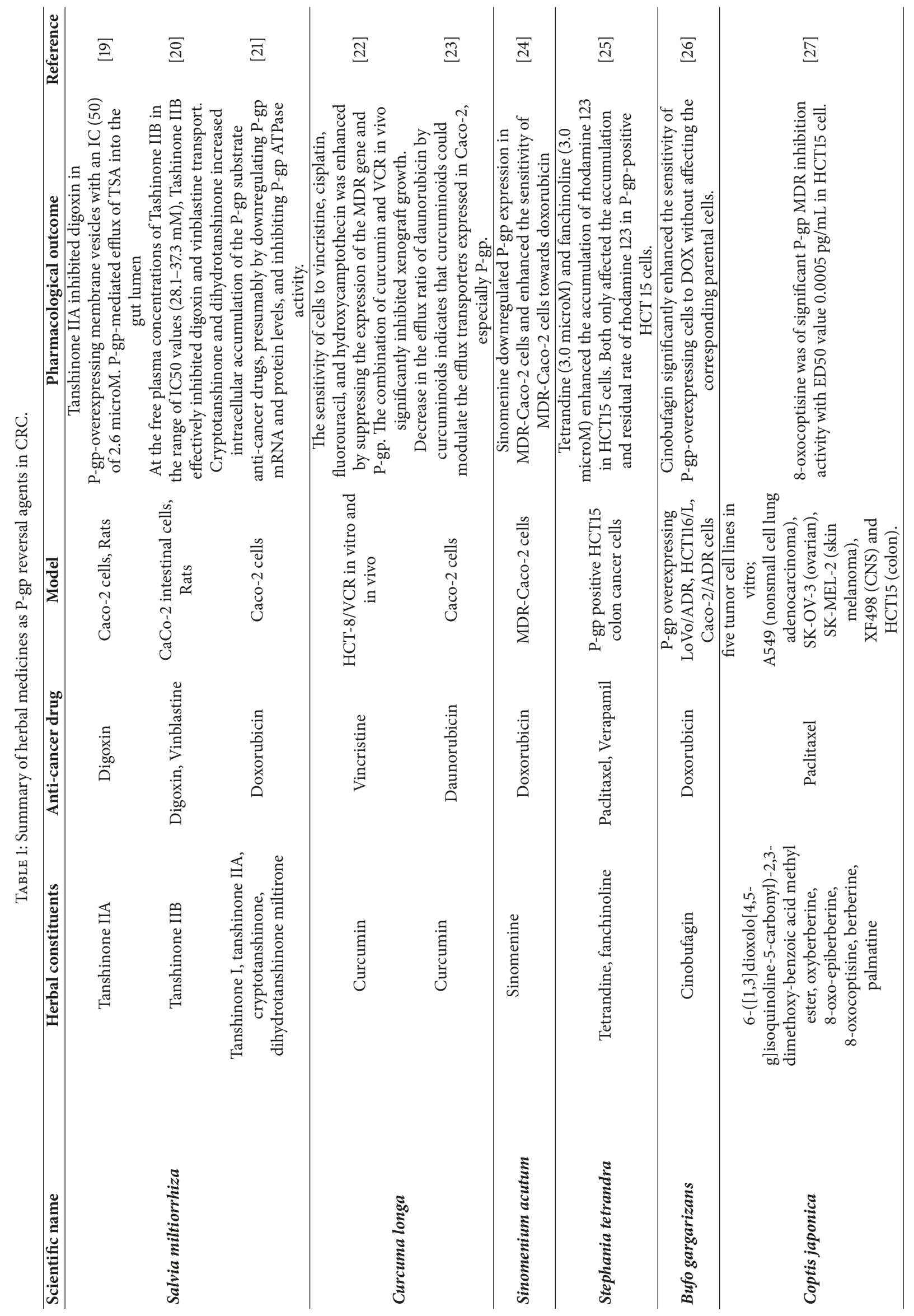




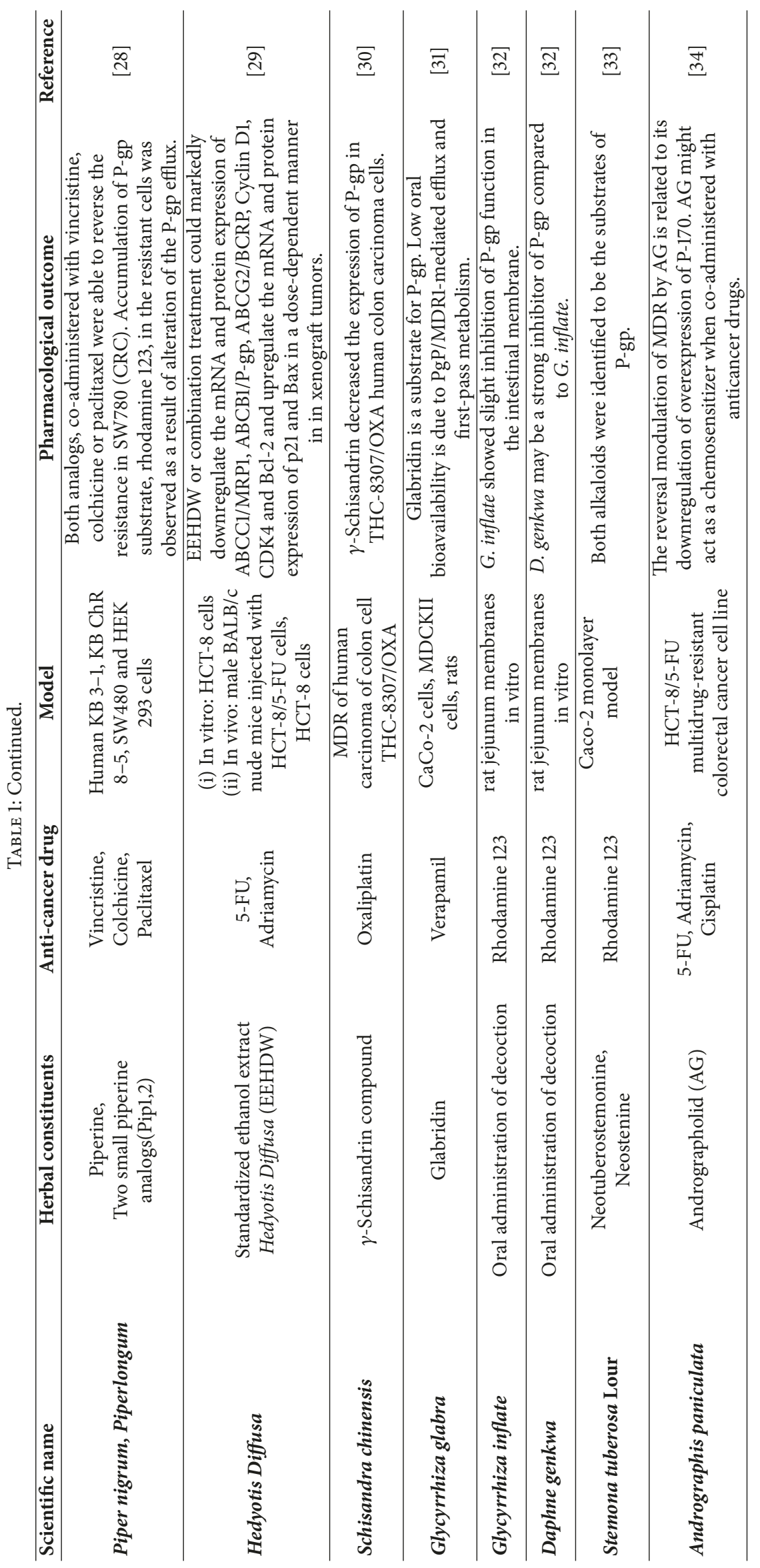


dihydrotanshinone, and miltirone) on doxorubicin (DOX) and irinotecan efflux in Caco-2 cells. They found that two tanshinones in particular, cryptotanshinone and dihydrotanshinone, downregulated P-gp mRNA and protein expression and inhibited P-gp ATPase activity, thereby increasing intracellular accumulation of P-gp-substrate anticancer drugs [21].

3.2. C. longa Rhizome. Curcumin, extracted from the rhizomes of C. longa, is well known as a major component of curry dishes in many Asian countries. Curcumin has been used not only for cooking but also in traditional medicine. It is known to be useful in various conditions, including cardiovascular health, cognitive function, and cancer treatment [36-38].

Curcumin has also been proposed as a supportive medicine in the chemotherapy of CRC. At concentrations greater than $25 \mu \mathrm{M}$, curcumin was shown to enhance the sensitivity of human colon cells to treatment with vincristine, cisplatin, fluorouracil, and hydroxycamptothecin by suppressing the expression of the MDR gene and P-gp in vitro. The combination of curcumin and vincristine also significantly inhibited xenograft growth in vivo by reducing expression of MDR1 [22]. A subsequent study in 2010 testing extracts from rhizomes of $C$. longa and Curcuma spp. on Caco- 2 cells showed increases in intracellular accumulation of the anticancer agent rhodamine 123 (R123) in both parental Caco- 2 cells and vinblastine-selected Caco- 2 cells. Moreover, these curcuminoids downregulated P-gp expression in drugresistant Caco-2 cells, as evidence by a significant decrease in the efflux ratio of daunorubicin [23]. Tumerones, another class of sesquiterpenoids isolated from C. longa, were found to facilitate transport of curcumin into intestinal Caco-2 cells and enhance inhibition of P-gp activity, suggesting the potential of this agent to improve absorption of curcumin in the intestine [39].

3.3. S. acutum and S. tetrandra Rhizome. The rhizome of $S$. acutum and Stephania tetrandra (Chinese name, Fang ji) has been used as a folk remedy for rheumatic and arthritic diseases and neuralgia [40, 41]. In a recent study, sinomenine, extracted from the stem of $S$. acutum, was found to enhance the sensitivity of CRC cells to DOX [24]. Specifically, sinomenine at a concentration of $500 \mu \mathrm{M}$, which did not decrease cell viability alone, increased the sensitivity of DOX-resistant MDR-Cacp-2 cells to added DOX. This action was associated with downregulation of MDR1 and COX2 (cyclooxygenase 2). Consistent with this latter effect, treatment of MDR-Caco-2 cells with sinomenine decreased the release of prostaglandin E2 (PGE2), which was significantly elevated in these cells compared with parental Caco2 cells. Furthermore, two bisbenzylisoquinoline alkaloids, tetrandrine and fangchinoline, extracted from S. tetrandra also showed to work as P-gp substrate in colon cancer cells. Choi et al. found that tetrandrine (3.0 microM) and fangchinoline ( 3.0 microM) increased the accumulation rates of rhodamine 123 in HCT cells whereas there was no effect on the cytotoxicity to P-gp negative cells [25].
3.4. B. gargarizans. Secretions from the skin and auricular glands of B. gargarizans (Asiatic toad) are called CahnSu in traditional Chinese medicine. Traditionally used against cardiac arrhythmias because of its cardiotonic effects [26], this secretion has recently been shown to have potential for cancer treatment. Bufadienolides-steroids isolated from the skin and parotid venom glands of the toad B. gargarizans-were shown to induce p53-mediated apoptosis in esophageal squamous cell carcinoma cells in vitro and in vivo [42]. In CRC cells, cinobufagin, another representative steroid, showed a reversal effect on P-gp-mediated MDR. Cinobufagin significantly enhanced the sensitivity of P-gp-overexpressing cells to DOX without affecting the corresponding parental cells. Studies of this agent further revealed that the mechanism of action involved noncompetitive inhibition of P-gp ATPase activity [26].

3.5. C. japonica Makino Rhizome. Haung Lian, the Chinese name for the rhizome of $C$. japonica, is widely used in various formulations for treating intestinal infection and inflammation; it is also known to possess central nervous system- (CNS-) depressant activities. The best known alkaloid in extracts of C. japonica is berberine, which has attracted considerable interest for its therapeutic potential in metabolic diseases [43]. More recently, Min et al. tested whether six components from C. japonica rhizome -6 -([1,3] dioxolo[4,5-g]isoquinoline-5-carbonyl)-2,3-dimethoxybenzoic acid methyl ester, oxyberberine, 8-oxo-epiberberine, 8 -oxocoptisine, berberine, and palmatine-increased the sensitivity of five tumor cell lines-A549 (lung adenocarcinoma), SK-OV-3 (ovarian), SK-MEL-2 (skin melanoma), XF498 (CNS), and HCT15 (colon) - to paclitaxel. Among the compounds tested, 8-oxocoptisine showed significant inhibitory activity against P-gp-mediated MDR in HCT15 cells [27].

3.6. P. nigrum and P. longum Fructus. P. nigrum (black pepper) is a common spice and food additive. The fruit of $P$. nigrum and P. longum (Chinese name, Bi bo) has been used in traditional medicine for its important medicinal and preservative properties in intestinal diseases. It is also included in many traditional formulas to enhance the activity of other bioactive compounds, such as curcumin [44]. Its anti-inflammatory, antimicrobial, antioxidant, and anticarcinogenic activities have been experimentally verified [45]. In an attempt to identify derivatives that better interact with Pgp than piperine, Syed et al. designed the piperine analogs, Pip 1 and Pip 2, in silico and tested them in vitro. These latter experiments demonstrated that both analogs were better able to reverse the resistance to vincristine, colchicine, or paclitaxel in KB (cervical) and SW480 (colon) cancer cell lines than piperine. In support of these findings, these researchers further showed that Pip 1 and Pip 2 increased accumulation of the P-gp substrate R123 in these drugresistant cells [28].

3.7. H. diffusa Herba. H. diffusa is a slender, annual plant that is widely distributed throughout Asia. The whole herb of $H$. diffusa, named Bai Hua She She Cao in Chinese, 
has traditionally been used to treat inflammation, urethral infection, contusions, and ulcers. Ye et al. found that an aqueous extract of $H$. diffusa exerted protective effects against renal inflammation in vivo [46]. In addition, an ethanol extract of $H$. diffusa was shown to increase the sensitivity of HCT-8/D-FU cells to 5-fluoruricil (5-FU) by inhibiting drug efflux and thereby increasing intracellular accumulation of 5-FU. Notably, this latter extract was shown to significantly downregulate mRNA and protein expression of drug efflux pumps, including ABCC1/MRP1 and ABCB1/P-gp [29].

3.8. S. chinensis Fructus. The fruit of S. chinensis is a medicinal herb whose Chinese name, Wu wei zi, means containing five flavors: salty, sweet, sour, spicy, and bitter. The active components of $S$. chinensis include deoxyschisandrin, $\gamma$-schisandrin, schizandrin, and gomisin A [47]. Numerous investigations of the effects of $S$. chinensis on hepatic damage have been conducted in recent decades [48, 49]. A comparison of the effects of $S$. chinensis extract and its lignin components gomisin $\mathrm{A}$, gomisin $\mathrm{N}$, and schizandrin $\mathrm{C}$ in Caco- 2 cell lines showed that the transport of gomisin $\mathrm{N}$ is mediated by the MRP transporter. This indicates that gomisin $\mathrm{N}$ may bind competitively to MRP and P-gp [50]. In addition, $\gamma$-schizandrin was subsequently shown to exert an MDRreversal effect in THC-8307/OXA human colon carcinoma cells by decreasing the expression of P-gp [30].

3.9. G. glabra, G. inflate Rhizome, and D. genkwa Flos. The root of G. glabra and G. inflate (Chinese name, Gancao) would be the most popular herbal medicine involved in approximately over $70 \%$ of herbal complex formulas. It was widely used for the bronchial remedies, gastrointestinal remedies, and mollification of toxicity traditionally. One of the main flavonoid extracted from G. glabra is glabridin, exhibiting cytotoxic activity, antimicrobial activity, etc. [51]. Cao et al. found that glabridin is a substrate for P-gp from the result that glabridin (2.56 microM) inhibited P-gp mediated transport of digoxin, but stimulating P-gp/MDR 1 ATPase activity. In addition, the systemic bioavailability of glabridin increased when combined with verapamil [31]. The oral administration of $G$. inflate was also found to inhibit P-gp in the intestinal membrane of rats slightly. In the same study, $D$. genkwa (Chinese name, Yan hua) decoction showed stronger P-gp inhibition effect than G. inflate and it could also decrease the permeability of secretory transport of rhodamine 123 across the jejunum tissues. Furthermore, combination of G. inflate and D. genkwa more significantly inhibited P-gp function compared to single administration of each herb [32].

3.10. S. tuberosa Lour Rhizome. Bai bu gen, the root of Stemonaceae family, was used in the treatment of respiratory diseases and the anthelminthic for a thousand years, and several alkaloids from S. tuberosa were verified for their antitussive activity in 2003. [52] Neotuberostemonine and neostenine, two major alkaloids, were P-gp substrates with exhibiting good intestinal absorptions, which means it can be orally active components. In addition, verapamil and cyclosporine A, P-gp inhibitors, resulted in decrease in secretory transport of two alkaloids and enhancement of absorptive transport of both alkaloids [33].

3.11. A. paniculata Herba. A. paniculata is a traditional herb common in Southeast Asia and found from India to China. It is known for the hypotensive effect and used for symptomatic relief of respiratory tract infections $[53,54]$. Along with traditional usage, Ajaya et al. found that the methanolic extract of A. paniculata, especially dichloromethane fraction, exhibited anticancer and immunomodulatory activities by inhibiting proliferation of HT-29(colon cancer cell) and proliferating human peripheral blood lymphocytes [55]. Andrographolide, another major component extracted from A. paniculata, might modulate MDR by downregulating the overexpression of P-170 on HCT-8/5-FU cell line. The low concentration of andrographolide has no significant effectiveness, whereas it appeared to be effective when coadministered with 5-FU, Adriamycin, and cisplatin [34]. It implies the possibility of $A$. paniculata as chemosensitizer with anticancer drugs in colon cancer.

\section{Discussion}

MDR is a major obstacle that severely limits the efficacy of clinical chemotherapy, especially in the treatment of CRC. Among several mechanisms related to MDR, P-gp mediated MDR takes a significant part pathologically. The brief mechanism of P-gp mediated MDR is as follows: the mechanism of drug efflux reflects the structural transition of P-gp, which starts with dimerization of the NBDs to form an ATP-binding pocket. This allows the binding of compounds to P-gp. Upon binding of the substrate to the high-affinity drug-binding sites formed by 12 TMHs in the internal surface, the TMDs undergo a conformational change, switching from an inward to outward orientation in association with efflux of the substrate to the extracellular environment. ATP hydrolysis provides the energy for the dissociation of NBDs and resetting of the initial P-gp conformation [56]. Notably, most drugs used in chemotherapy for CRC, including targeted agents, are substrates of P-gp and thus show diminished intracellular accumulation $[2,57]$.

Since the first effort in 1981 by Tsuruo et al. [58], many attempts have been made to identify effective Pgp inhibitors. First-generation inhibitors were originally developed for other indications but were discovered to inhibit P-gp. Other examples of early-discovered inhibitors include immunosuppressants such as cyclosporin $\mathrm{A}$; the antihypertensives, reserpine, quinidine and yohimbine; and antiestrogens, including tamoxifen and toremifene [9]. Many first-generation compounds are themselves substrates for Pgp and compete with coadministered substrates for efflux. As a result, high doses of these agents were required to have an impact on CRC. Because of their high cellular toxicity and nonselective effects, their use has been limited and led to the development of second-generation compounds [59]. To compensate for the defects of first-generation agents, which have low potency and specificity and high cellular toxicity, researchers structurally modified first-generation 
inhibitors, yielding second-generation compounds. Representative second-generation inhibitors include compounds such as PSC 833, a nonimmunosuppressive analog of cyclosporin A; dexverapamil, the R-isomer of verapamil lacking cardiac effects; and biricodar, also known as VX$710[59,60]$. Despite improved performance, these agents could still not be used clinically, at least in part because most second-generation inhibitors also inhibited cytochrome 3A4 (CYP3A4). This undesirable action reflects the competition between anticancer agents and MDR modulators for CYP3A4 activity, which led to unpredictable interactions with anticancer drugs and reduced the bioavailable drug to subtoxic levels [61]. Third-generation inhibitors were designed to be greater than 200-fold more potent than first- and secondgeneration compounds and show almost no pharmacological interactions with other drugs. Third-generation compounds such as tariquidar (XR9576), zosuquidar (LY335979), laniquidar (R101933), and elacridar (F12091) are currently awaiting clinical trials as P-gp inhibitors [62].

After the repeated clinical failures of the established resistance-modifying agents, researches seeking P-gp inhibitors from the natural products had the limelight. In this study, we reviewed 16 studies demonstrating 14 herbal medicines-S. miltiorrhiza, C. longa, S. acutum, S. tetrandra, B. gargarizans, C. japonica, $P$. nigrum and P. longum, $H$. diffusa, S. chinensis, G. glabra, G. inflate and D. genkwa, S. tuberosa Lour, and A. paniculata - that have been demonstrated to alter P-gp-mediated drug efflux in CRC. Most of these studies were conducted in vitro; only two studies using C. longa and $H$. Diffusa were conducted in vivo, but none were performed in humans. Among the reviewed studies, the most frequently used CRC cellular line was Caco-2 cell. As it develops microvilli on the apical surface and expresses intestinal transporters such as P-gp, it has been widely used model for study of transport characteristics of drugs from introduction in the early 1990s. $[63,64]$ These 14 herbal medicines showed clear effects against CRC cells that were resistant to specific anticancer drugs, including doxorubicin, daunorubicin, vinblastine, vincristine, paclitaxel, verapamil, 5-FU, and oxaliplatin. The mechanism of herbal medicines against MDR mediated by P-gp can be summarized into two, acting as a P-gp substrate or downregulating P-gp expression in cellular membranes.

The limitation of our review pertains to the paucity of data. Further investigations in a clinical setting and more detailed mechanistic studies are warranted. Also, the fact that agents that modulate MDR may not act by directly inhibiting P-gp should also be taken into account. Our decision to exclude natural products, not used as herbal medicine, might also be criticized. However, we strongly feel that rather than listing a vast store of natural plants, proposing the practical herbal medicines could give a cue to further progress.

Above all, we observantly investigated natural products which were used as traditional medicine in East Asia. Since these herbal medicines have been used for thousands of years until nowadays, there are large amount of accumulated information of each products. An advantage of herbal medicine is that they function as multicomponent to multitarget (MCMT) agents. Thus, the overall potency/efficacy of herbal medicines cannot be defined by the action of a single compound derived from the herb. In addition to their MCMT mechanism, herbal medicines are expected to exhibit low side effects, good tolerability, and additional anticancer pharmacological actions. These properties may allow herbal medicine to be used as an effective adjunct to chemotherapy in CRC.

\section{Conclusions}

In this review, fourteen natural products that have been used in traditional medicine everlastingly were highlighted as potential P-gp reversal agents in CRC. It is expected that additional investigations of natural herbs will establish a crucial role for these agents in the successful chemotherapy of CRC.

\section{Conflicts of Interest}

There are no conflicts of interest.

\section{Acknowledgments}

This work was supported by a grant from the National Research Foundation of Korea (NRF) funded by the Korean government (Ministry of Science and ICT) (no. 2017R1C1B5076590).

\section{References}

[1] R. L. Siegel, K. D. Miller, S. A. Fedewa et al., "Colorectal cancer statistics," CA: A Cancer Journal for Clinicians, vol. 67, no. 3, pp. 177-193, 2017.

[2] A. T. Fojo, K. Ueda, D. J. Slamon, D. G. Poplack, M. M. Gottesman, and I. Pastan, "Expression of a multidrug-resistance gene in human tumors and tissues," Proceedings of the National Acadamy of Sciences of the United States of America, vol. 84, no. 1, pp. 265-269, 1987.

[3] R. Krishna and L. D. Mayer, "Multidrug resistance (MDR) in cancerMechanisms, reversal using modulators of MDR and the role of MDR modulators in influencing the pharmacokinetics of anticancer drugs," European Journal of Pharmaceutical Sciences, vol. 11, no. 4, pp. 265-283, 2000.

[4] S. Chai, K. K. To, and G. Lin, "Circumvention of multi-drug resistance of cancer cells by Chinese herbal medicines," Chinese Medicine, vol. 5, no. 1, p. 26, 2010.

[5] V. Goler-Baron, I. Sladkevich, and Y. G. Assaraf, "Inhibition of the PI3K-Akt signaling pathway disrupts ABCG2-rich extracellular vesicles and overcomes multidrug resistance in breast cancer cells," Biochemical Pharmacology, vol. 83, no. 10, pp. 1340-1348, 2012.

[6] D. B. Longley and P. G. Johnston, "Molecular mechanisms of drug resistance," The Journal of Pathology, vol. 205, no. 2, pp. 275-292, 2005.

[7] M. M. Gottesman, "Mechanisms of cancer drug resistance," Annual Review of Medicine, vol. 53, pp. 615-627, 2002.

[8] G.-T. Ho, F. M. Moodie, and J. Satsangi, "Multidrug resistance 1 gene (P-glycoprotein 170): An important determinant in gastrointestinal disease?" Gut, vol. 52, no. 5, pp. 759-766, 2003. 
[9] T. Bansal, M. Jaggi, R. K. Khar, and S. Talegaonkar, "Emerging significance of flavonoids as P-glycoprotein inhibitors in cancer chemotherapy," Journal of Pharmacy \& Pharmaceutical Sciences, vol. 12, no. 1, pp. 46-78, 2009.

[10] K. Ellis, J. W. Marlin, T. A. H. Taylor et al., “The effects of human immunodeficiency virus infection on the expression of the drug efflux proteins P-glycoprotein and breast cancer resistance protein in a human intestine model," Journal of Pharmacy and Pharmacology, vol. 67, no. 2, pp. 178-188, 2015.

[11] F. Thiebaut, T. Tsuruo, H. Hamada, M. M. Gottesman, I. Pastan, and M. C. Willingham, "Cellular localization of the multidrug-resistance gene product P-glycoprotein in normal human tissues," Proceedings of the National Acadamy of Sciences of the United States of America, vol. 84, no. 21, pp. 7735-7738, 1987.

[12] U. Potočnik, M. Ravnik-Glavač, R. Golouh, and D. Glavač, "Naturally occurring mutations and functional polymorphisms in multidrug resistance 1 gene: Correlation with microsatellite instability and lymphoid infiltration in colorectal cancers [4]," Journal of Medical Genetics, vol. 39, no. 5, pp. 340-346, 2002.

[13] T. Yamada, A. S. Takaoka, and Y. Naishiro, "Transactivation of the Multidrug Resistance 1 Gene by T-Cell Factor 4/(beta)Catenin Complex in Early Colorectal Carcinogenesis," Cancer Research, vol. 60, no. 17, pp. 4761-4766, 2000.

[14] R. L. Juliano and V. Ling, "A surface glycoprotein modulating drug permeability in Chinese hamster ovary cell mutants," Biochimica et Biophysica Acta, vol. 455, no. 1, pp. 152-162, 1976.

[15] L. Esser, F. Zhou, K. M. Pluchino et al., "Structures of the Multidrug Transporter P-glycoprotein Reveal Asymmetric ATP Binding and the Mechanism of Polyspecificity," The Journal of Biological Chemistry, vol. 292, no. 2, pp. 446-461, 2017.

[16] A. K. Tiwari, K. Sodani, C.-L. Dai, C. R. Ashby Jr., and Z.-S. Che, "Revisiting the ABCs of multidrug resistance in cancer chemotherapy," Current Pharmaceutical Biotechnology, vol. 12, no. 4, pp. 570-594, 2011.

[17] T. Eichhorn and T. Efferth, "P-glycoprotein and its inhibition in tumors by phytochemicals derived from Chinese herbs," Journal of Ethnopharmacology, vol. 141, no. 2, pp. 557-570, 2012.

[18] Z. Wang, C. Xie, Y. Huang, C. W. K. Lam, and M. S. S. Chow, "Overcoming chemotherapy resistance with herbal medicines: Past, present and future perspectives," Phytochemistry Reviews, vol. 13, no. 1, pp. 323-337, 2014.

[19] M. Gu, G. Zhang, Z. Su, and F. Ouyang, "Identification of major active constituents in the fingerprint of Salvia miltiorrhiza Bunge developed by high-speed counter-current chromatography," Journal of Chromatography A, vol. 1041, no. 1-2, pp. 239243, 2004.

[20] X.-Y. Yu, S.-G. Lin, Z.-W. Zhou et al., "Role of P-glycoprotein in the intestinal absorption of tanshinone IIA, a major active ingredient in the root of Salvia miltiorrhiza Bunge," Current Drug Metabolism, vol. 8, no. 4, pp. 325-340, 2007.

[21] T. Hu, K. K. W. To, L. Wang et al., "Reversal of P-glycoprotein (Pgp) mediated multidrug resistance in colon cancer cells by cryptotanshinone and dihydrotanshinone of Salvia miltiorrhiza," Phytomedicine, vol. 21, no. 11, pp. 1264-1272, 2014.

[22] W.-D. Lu, Y. Qin, C. Yang, L. Li, and Z.-X. Fu, "Effect of curcumin on human colon cancer multidrug resistance in vitro and in vivo," Clinics, vol. 68, no. 5, pp. 694-701, 2013.

[23] C. Ampasavate, U. Sotanaphun, P. Phattanawasin, and N. Piyapolrungroj, "Effects of Curcuma spp. on P-glycoprotein function," Phytomedicine, vol. 17, no. 7, pp. 506-512, 2010.
[24] Z. Liu, Z.-J. Duan, J.-Y. Chang et al., "Sinomenine sensitizes multidrug-resistant colon cancer cells (Caco-2) to doxorubicin by downregulation of MDR-1 expression," PLoS ONE, vol. 9, no. 6, Article ID e98560, 2014.

[25] S.-U. Choi, S.-H. Park, K.-H. Kim et al., "The bisbenzylisoquinoline alkaloids, tetrandine and fangchinoline, enhance the cytotoxicity of multidrug resistance-related drugs via modulation of P-glycoprotein," Anti-Cancer Drugs, vol. 9, no. 3, pp. 255261, 1998.

[26] Z. Yuan, X. Shi, Y. Qiu et al., "Reversal of P-gp-mediated multidrug resistance in colon cancer by cinobufagin," Oncology Reports, vol. 37, no. 3, pp. 1815-1825, 2017.

[27] Y. D. Min, M. C. Yang, K. H. Lee, K. R. Kim, S. U. Choi, and K. R. Lee, "Protoberberine alkaloids and their reversal activity of P-gp expressed multidrug resistance (MDR) from the rhizome of Coptis japonica Makino," Archives of Pharmacal Research, vol. 29, no. 9, pp. 757-761, 2006.

[28] S. B. Syed, H. Arya, I. Fu et al., "Targeting P-glycoprotein: Investigation of piperine analogs for overcoming drug resistance in cancer," Scientific Reports, vol. 7, no. 1, 2017.

[29] L. Jiumao, The Effect and Mechanism of Hedyotis Diffusa Willd Reverse Multi-drug Resistance in Colorectal Cancer [Ph.D. thesis], Chinese Medicine, Fujian University, 2016.

[30] X. D. Jin, Q. L. Xu, and X. Z. Zheng, "Reversal Effect of ?Schisandrin Compound on Multidrug Resistance of Human Carcinoma of Colon Cell THC-8307," Med. Recapitul, vol. 10, pp. 3-5, 2011.

[31] J. Cao, X. Chen, J. Liang et al., "Role of P-glycoprotein in the intestinal absorption of glabridin, an active flavonoid from the root of Glycyrrhiza glabra," Drug Metabolism and Disposition, vol. 35, no. 4, pp. 539-553, 2007.

[32] B.-B. Huang, G.-F. Li, F. Ren et al., "Effect of Glycyrrhiza inflata and Daphne genkwa on permeabilities of rhodamine 123, a Pglycoprotein substrate across rat jejunum membranes in vitro," Zhongguo Zhong Yao Za Zhi, vol. 33, no. 21, pp. 2521-2526, 2008.

[33] P. H. H. Leung, L. Zhang, Z. Zuo, and G. Lin, "Intestinal absorption of Stemona alkaloids in a Caco-2 cell model," Planta Medica, vol. 72, no. 3, pp. 211-216, 2006.

[34] Y. Han, L. M. Bu, X. Ji, C. Y. Liu, and Z. H. Wang, "Modulation of multidrug resistance by andrographolid in a HCT-8/5FU multidrug-resistant colorectal cancer cell line," Journal of Digestive Diseases, vol. 6, no. 2, pp. 82-86, 2005.

[35] X.-Y. Yu, Z.-W. Zhou, S.-G. Lin et al., "Role of ATP-binding cassette drug transporters in the intestinal absorption of tanshinone IIB, one of the major active diterpenoids from the root of Salvia miltiorrhiza," Xenobiotica, vol. 37, no. 4, pp. 375-415, 2007.

[36] U. Kukongviriyapan, K. Apaijit, and V. Kukongviriyapan, "Oxidative stress and cardiovascular dysfunction associated with cadmium exposure: Beneficial effects of curcumin and tetrahydrocurcumin," The Tohoku Journal of Experimental Medicine, vol. 239, no. 1, pp. 25-38, 2016.

[37] R. Sinha, D. E. Anderson, S. S. McDonald, and P. Greenwald, "Cancer risk and diet in India," J. Postgrad. Med, vol. 49, no. 3, pp. 222-228, 2003.

[38] J. Choi, C. Oh, S. Shim, S. Jeong, H. S. Kim, and M. Kim, "Reduction in Prevalence of Hypertension and Blood Heavy Metals among Curry-Consumed Korean," The Tohoku Journal of Experimental Medicine, vol. 244, no. 3, pp. 219-229, 2018.

[39] G. G. L. Yue, S.-W. Cheng, H. Yu et al., "The role of turmerones on curcumin transportation and P-glycoprotein activities in 
intestinal caco-2 cells," Journal of Medicinal Food, vol. 15, no. 3, pp. 242-252, 2012.

[40] H. Yamasaki, "Pharmacology of sinomenine, an anti-rheumatic alkaloid from Sinomenium acutum," Acta Medica Okayama, vol. 30, no. 1, pp. 1-20, 1976.

[41] K. Chan, Z. Q. Liu, Z. H. Jiang et al., "The effects of sinomenine on intestinal absorption of paeoniflorin by the everted rat gut sac model," Journal of Ethnopharmacology, vol. 103, no. 3, pp. 425-432, 2006.

[42] S. Lin, J. Lv, P. Peng et al., "Bufadienolides induce p53-mediated apoptosis in esophageal squamous cell carcinoma cells in vitro and in vivo," Oncology Letters, vol. 15, no. 2, pp. 1566-1572, 2018.

[43] J. Lan, Y. Zhao, F. Dong et al., "Meta-analysis of the effect and safety of berberine in the treatment of type 2 diabetes mellitus, hyperlipemia and hypertension," Journal of Ethnopharmacology, vol. 161, pp. 69-81, 2015.

[44] M. Meghwal and T. K. Goswami, "Piper nigrum and piperine: An update," Phytotherapy Research, vol. 27, no. 8, pp. 1121-1130, 2013.

[45] S. Darshan and R. Doreswamy, "Patented antiinflammatory plant drug development from traditional medicine," Phytotherapy Research, vol. 18, no. 5, pp. 343-357, 2004.

[46] J.-H. Ye, M.-H. Liu, X.-L. Zhang, and J.-Y. He, "Chemical profiles and protective effect of Hedyotis diffusa willd in lipopolysaccharide-induced renal inflammation mice," International Journal of Molecular Sciences, vol. 16, no. 11, pp. 2725227269, 2015.

[47] Z. Yao, X. Liu, and Y. Gu, "Schisandra chinensis Baill, a Chinese medicinal herb, alleciates high-fat-diet-including nonalcoholic steatohepatitis in rats," African Journal of Traditional, Complementary and Alternative Medicines, vol. 11, no. 1, pp. 222227, 2014.

[48] C. Liu, S. Zhang, and H. Wu, "Non-thermal extraction of effective ingredients from Schisandra chinensis baill and the antioxidant activity of its extract," Natural Product Research (Formerly Natural Product Letters), vol. 23, no. 15, pp. 1390-1401, 2009.

[49] Q. Chen, Y. J. Wu, N. N. Cheng et al., "Dual effects of extract of Schisandra chinensis Baill on rat hepatic CYP3A," Acta Pharmaceutica Sinica B, vol. 45, no. 9, pp. 1194-1198, 2010.

[50] V. L. M. Madgula, B. Avula, Y. W. Choi et al., "Transport of Schisandra chinensis extract and its biologically-active constituents across Caco-2 cell monolayers-an in-vitro model of intestinal transport," Journal of Pharmacy \& Pharmacology, vol. 60, no. 3, pp. 363-370, 2008.

[51] T. Fukai, K. Satoh, T. Nomura, and H. Sakagami, "Preliminary evaluation of antinephritis and radical scavenging activities of glabridin from Glycyrrhiza glabra," Fitoterapia, vol. 74, no. 7-8, pp. 624-629, 2003.

[52] H.-S. Chung, P.-M. Hon, G. Lin, P. P.-H. But, and H. Dong, "Antitussive Activity of Stemona Alkaloids from Stemona tuberosa," Planta Medica, vol. 69, no. 10, pp. 914-920, 2003.

[53] R. Subramanian, M. Z. Asmawi, and A. Sadikun, "In vitro $\alpha$-glucosidase and $\alpha$-amylase enzyme inhibitory effects of Andrographis paniculata extract and andrographolide," Acta Biochimica Polonica, vol. 55, no. 2, pp. 391-398, 2008.

[54] X.-Y. Hu, R.-H. Wu, M. Logue et al., "Andrographis paniculata (Chuān Xīn Lián) for symptomatic relief of acute respiratory tract infections in adults and children: A systematic review and meta-analysis," PLoS ONE, vol. 12, no. 8, 2017.
[55] R. A. Kumar, K. Sridevi, N. V. Kumar, S. Nanduri, and S. Rajagopal, "Anticancer and immunostimulatory compounds from Andrographis paniculata," Journal of Ethnopharmacology, vol. 92, no. 2-3, pp. 291-295, 2004.

[56] S. Wilkens, "Structure and mechanism of ABC transporters," F1000Prime Reports, vol. 7, pp. 1-9, 2015.

[57] S. Meschini, A. Calcabrini, E. Monti et al., "Intracellular Pglycoprotein expression is associated with the intrinsic multidrug resistance phenotype in human colon adenocarcinoma cells," International Journal of Cancer, vol. 87, no. 5, pp. 615-628, 2000.

[58] T. Tsuruo, H. Iida, S. Tsukagoshi, and Y. Sakurai, "Overcoming of vincristine resistance in P388 leukemia in vivo and in vitro through enhanced cytotoxicity of vincristine and vinblastine by verapamil," Cancer Research, vol. 41, no. 5, pp. 1967-1972, 1981.

[59] A. H. Dantzig, D. P. De Alwis, and M. Burgess, "Considerations in the design and development of transport inhibitors as adjuncts to drug therapy," Advanced Drug Delivery Reviews, vol. 55, no. 1, pp. 133-150, 2003.

[60] M. V. S. Varma, Y. Ashokraj, C. S. Dey, and R. Panchagnula, "P-glycoprotein inhibitors and their screening: a perspective from bioavailability enhancement," Pharmacological Research, vol. 48, no. 4, pp. 347-359, 2003.

[61] H. Thomas and H. M. Coley, "Overcoming multidrug resistance in cancer: an update on the clinical strategy of inhibiting Pglycoprotein," Cancer Control, vol. 10, no. 2, pp. 159-165, 2003.

[62] W. Li, H. Zhang, Y. G. Assaraf et al., "Overcoming ABC transporter-mediated multidrug resistance: Molecular mechanisms and novel therapeutic drug strategies," Drug Resistance Updates, vol. 27, pp. 14-29, 2016.

[63] P. Artursson, K. Palm, and K. Luthman, "Caco-2 monolayers in experimental and theoretical predictions of drug transport," Advanced Drug Delivery Reviews, vol. 22, no. 1-2, pp. 67-84, 1996.

[64] V. Meunier, M. Bourrié, Y. Berger, and G. Fabre, “The human intestinal epithelial cell line Caco-2; pharmacological and pharmacokinetic applications," Cell Biology and Toxicology, vol. 11, no. 3-4, pp. 187-194, 1995. 


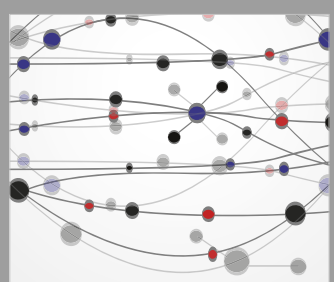

The Scientific World Journal
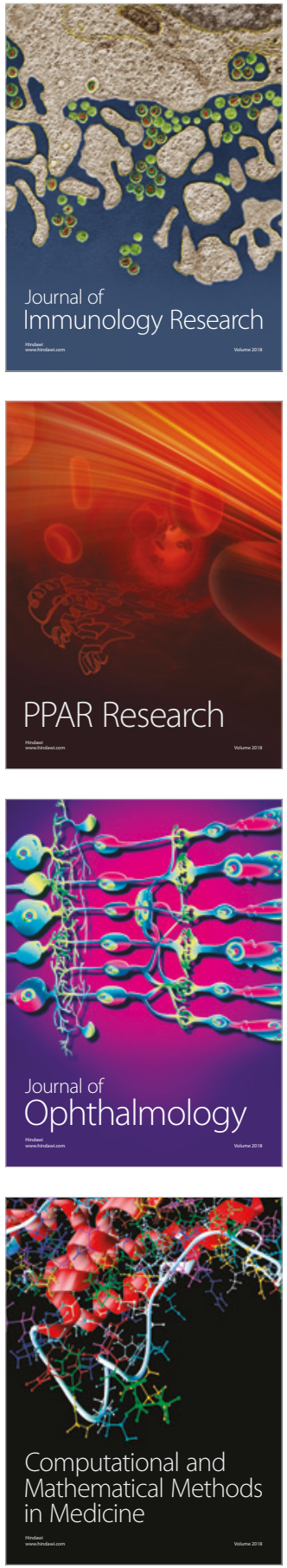

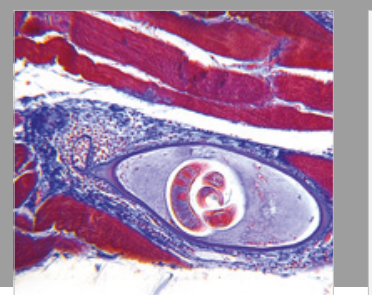

Gastroenterology Research and Practice

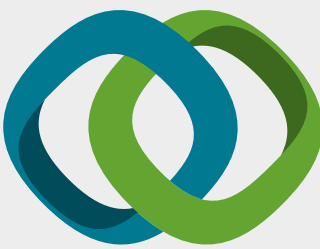

\section{Hindawi}

Submit your manuscripts at

www.hindawi.com
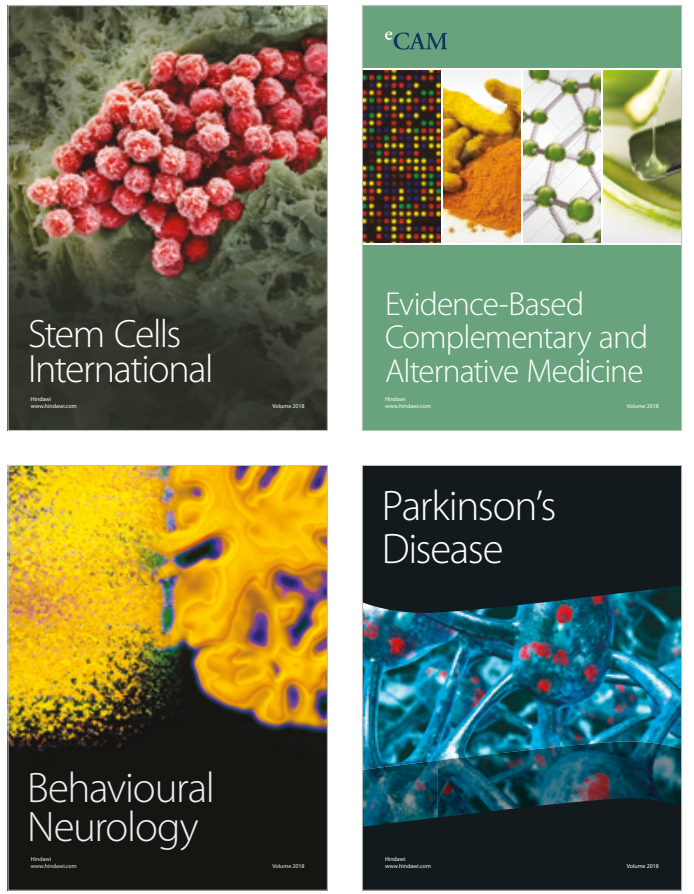

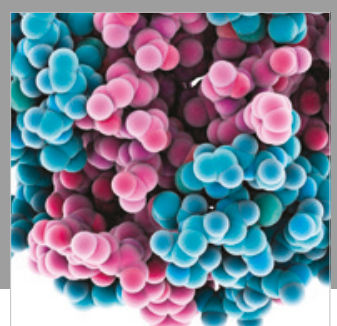

ournal of

Diabetes Research

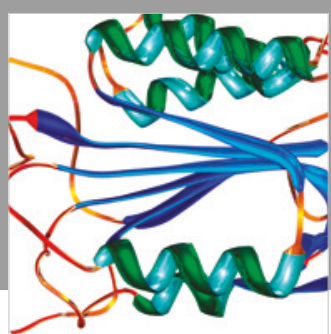

Disease Markers
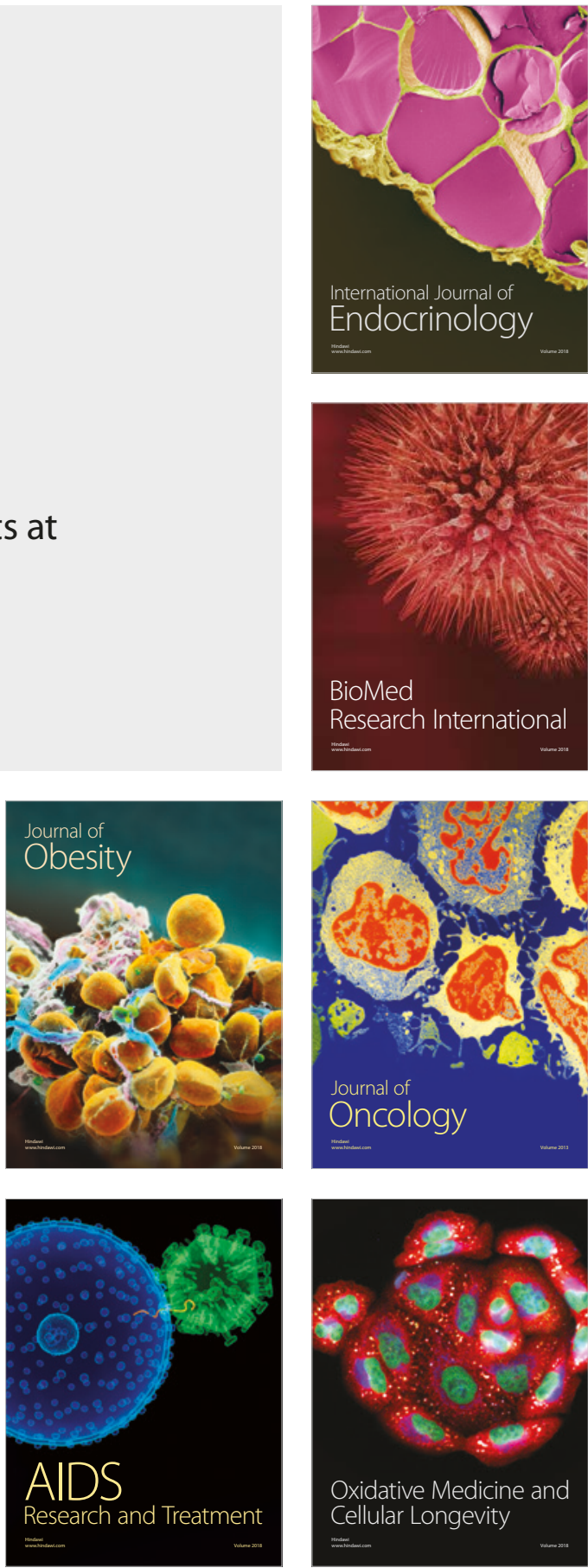